\title{
Dynamic evolution of COVID-19 on chest computed tomography: experience from Jiangsu Province of China
}

\author{
Yuan-Cheng Wang ${ }^{1} \cdot$ Huanyuan Luo ${ }^{2} \cdot$ Songqiao Liu $^{3} \cdot$ Shan Huang ${ }^{1} \cdot$ Zhen Zhou ${ }^{4} \cdot$ Qian $\mathrm{Yu}^{1} \cdot$ Shijun Zhang ${ }^{1}$. \\ Zhen Zhao ${ }^{1}$ - Yizhou $\mathrm{Yu}^{5}$. Yi Yang ${ }^{3}$. Duolao Wang ${ }^{2}$ - Shenghong Ju ${ }^{1}$ (b)
}

Received: 4 April 2020 /Revised: 30 April 2020 / Accepted: 20 May 2020 / Published online: 10 June 2020

(C) European Society of Radiology 2020

\begin{abstract} coronavirus disease 2019 (COVID-19) cohort in Jiangsu Province, China. was investigated from symptom onset (day 1 ) to beyond day 15. Comparison was made between severity groups. the course of illness.

Key Points

- Volume, density, and location of the pulmonary opacity on CT change over time in COVID-19.

- The evolution of CT appearance follows specific pattern, varying with disease severity.
\end{abstract}

Objectives To determine the patterns of chest computed tomography (CT) evolution according to disease severity in a large

Methods This retrospective cohort study was conducted from January 10, 2020, to February 18, 2020. All patients diagnosed with COVID-19 in Jiangsu Province were included, retrospectively. Quantitative CT measurements of pulmonary opacities including volume, density, and location were extracted by deep learning algorithm. Dynamic evolution of these measurements

Results A total of 484 patients (median age of 47 years, interquartile range 33-57) with $954 \mathrm{CT}$ examinations were included, and each was assigned to one of the three groups: asymptomatic/mild $(n=63)$, moderate $(n=378)$, severe/critically ill $(n=43)$. Time series showed different evolution patterns of CT measurements in the groups. Following disease onset, posteroinferior subpleural area of the lung was the most common location for pulmonary opacities. Opacity volume continued to increase beyond 15 days in the severe/ critically ill group, compared with peaking on days $13-15$ in the moderate group. Asymptomatic/mild group had the lowest opacity volume which almost resolved after 15 days. The opacity density began to drop from day 10 to day 12 for moderately ill patients. Conclusions Volume, density, and location of the pulmonary opacity and their evolution on CT varied with disease severity in COVID-19. These findings are valuable in understanding the nature of the disease and monitoring the patient's condition during

Keywords Coronavirus $\cdot$ Multidetector computed tomography $\cdot$ Viral pneumonia

Electronic supplementary material The online version of this article (https://doi.org/10.1007/s00330-020-06976-6) contains supplementary material, which is available to authorized users.

Shenghong Ju

jsh0836@hotmail.com

1 Department of Radiology, Zhongda Hospital, School of Medicine, Southeast University, 87 Ding Jia Qiao Road, Nanjing 210009, China

2 Department of Clinical Sciences, Liverpool School of Tropical Medicine, Liverpool L3 5QA, UK

3 Department of Critical Care Medicine, Zhongda Hospital, School of Medicine, Southeast University, Nanjing 210009, China

4 School of Electronics Engineering and Computer Science, Peking University, Beijing, China

5 Department of Computer Science, The University of Hong Kong, Pok Fu Lam, Hong Kong

\section{Abbreviations}

AI Artificial intelligence

CI Confidence interval

COVID-19 Coronavirus disease 2019

CT Computed tomography

GGO Ground-glass opacity

ICU Intensive care unit

IQR Interquartile range

$\mathrm{SaO}_{2} \quad$ Arterial oxygen saturation

SD Standard deviation

VOI Volume of interest 


\section{Introduction}

At the end of 2019, an outbreak of a highly contagious viral pneumonia was reported in Wuhan, China, and the causative agent was confirmed to be a novel coronavirus, termed as COVID-19 by the World Health Organization [1]. The disease spread to all provinces in China and many countries overseas, leaving more than 400,000 people infected as of March 25, 2020 [2, 3]. Jiangsu Province has the fifth largest population in China and has reported more than 600 infected cases [4].

Computed tomography (CT) examination plays an important role in the evaluation of COVID-19. According to the 5th edition of diagnosis and treatment standards for novel coronavirus pneumonia published by the Chinese National Health Commission, CT examination is one of the three main diagnostic criteria. In Hubei Province, the clinical diagnosis of COVID-19 comprises only clinical and radiological proof, irrespective of the nucleic acid test result, thus highlighting the importance of CT imaging assessment [5].

Some typical radiological findings including subpleural ground-glass opacities (GGO) and consolidation have been reported as well as the dynamic evolution of these lesions [6-10]. However, these measurements were mainly based on visual evaluation, which are subjective and inaccurate. In addition, imaging evolution according to disease severity has rarely been reported, which we believe is critical for understanding the nature of the disease and monitoring disease change in a stratified manner.

In this retrospective study, quantitative assessment of imaging characteristics and dynamic evolution was evaluated. Comparison was made among patients with variable disease severity.

Fig. 1 Flowchart of the study

\section{Materials and methods}

This study was approved by the ethics committee of Zhongda Hospital (2020ZDSYLL013-P01 and 2020ZDSYLL019$\mathrm{P} 01$ ), and informed consent was waived due to the emergent event of the pandemic.

Clinical information and imaging data for all COVID-19 patients diagnosed in Jiangsu before February 18, 2020, were obtained, retrospectively from electronic medical records, established by the Department of Health, Jiangsu Province.

Patients with no clinical records or with missing, incomplete, or poor-quality chest $\mathrm{CT}$ images were excluded. Figure 1 shows the detailed flowchart of this study.

Based on the 5th edition of the guideline for the diagnosis and treatment of COVID-19, published by the National Health Commission of the People's Republic of China on February 8, 2020, patients were assigned into three groups, classified according to disease severity: asymptomatic/mild, moderate, or severe/critically ill. Patients were classified as (i) "asymptomatic" if they had no symptoms of disease, (ii) "mild" if they had mild clinical symptoms, but no imaging abnormality, and (iii) "moderate" if they had one or more symptoms (fever, cough, diarrhea, etc.), and imaging showed manifestations of pneumonia or (iv) "severe" if they had one of the following conditions: (a) respiratory distress presenting with respiratory rate $\geq 30$ beats/min, (b) mean oxygen saturation in resting state $\leq 93 \%$, (c) arterial blood oxygen partial pressure/oxygen concentration $\leq 300 \mathrm{mmHg}$. (v) Patients with either shock, respiratory failure requiring mechanical ventilation, or combined organ failure requiring admission

626 patients diagnosed with COVID-19 from 24 hospitals between January 10 and February 18, 2020 in Jiangsu, China

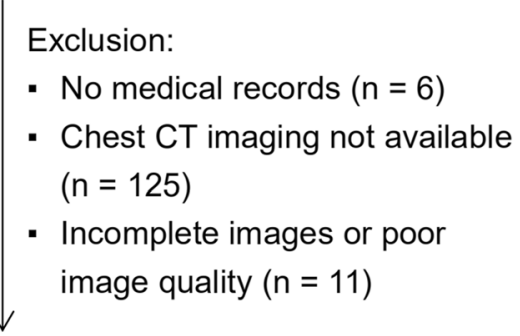

484 COVID-19 patients with 954 CT scans included

Lung segmentation and lesion

extraction by deep learning algorithm

Quantitative evaluation:

Volume / Density / Location / Dynamic evolution 
to an intensive care unit (ICU) were classified as "critically ill."

In order to analyze the evolution of imaging and clinical characteristics, the day when the initial symptoms emerged was defined as day 1 . When no initial symptoms were reported, the date of outpatient visit was used as day 1 . The time points for each CT scan, change of severity grading relative to day 1 , were recorded and were further assigned to days $1-3$, days 4-6, days 7-9, days 10-12, days 13-15, and beyond day 15 .

\section{CT acquisition}

CT imaging was performed using multislicer CT scanners. Thin-section images were collected preferentially (for further details relating to the acquisition of CT images, please see Table S1). All raw data with format of Digital Imaging and Communications in Medicine were then transferred to work stations for post-processing.

\section{Imaging analysis}

Lung segmentation, lesion extraction, and labeling were performed using a dedicated artificial intelligence (AI) system with deep learning algorithm for pulmonary pneumonia (Deepwise $\&$ League of PhD Technology Co., Ltd.). The AI algorithm achieved a dice of 0.97 in the HOUSE dataset (easy) and 0.89 in the LOLA dataset (hard), which proved its robustness. The accuracy of the AI system in lesion extraction and labeling was also manually checked by an investigator (Y.W.) with more than 10 years of experience in chest imaging. Bilateral lungs were segmented into the right upper lobe, right middle lobe, right lower lobe, left upper lobe, and left lower lobe automatically, and extraction of pulmonary opacities was carried out at the same time. All extracted opacity pieces were annotated as GGO or consolidation by the AI system and were approved by an investigator (Y.W.). The masks of opacities and each lung's lobes were saved for further voxel-based measurements including volume, X-ray attenuation, and location. Detailed information regarding segmentation and lesion extraction are available in supplemental methods.

Further assessment of segmented lobes and extracted opacities was performed as follows:

1. Volume measurement: it included volume of bilateral lungs, GGO, consolidation, GGO + consolidation, and aerated lung.

2. Density measurement: it included the X-ray attenuation of bilateral lungs, overall opacities, opacities in upper lobes including bilateral upper lobes and right middle lobe, and opacities in bilateral lower lobes.

3. Location analysis: A standard lung was selected from a healthy 37-year-old male without lung abnormalities. Subsequent registration and projection of all included chest
CT images to the standard lung was performed. Voxelbased frequencies of opacity were measured by drawing five volumes of interest (VOIs) in the standard lung, with each of the VOI larger than $15 \mathrm{~mL}$. Four VOIs were placed in the subpleural areas (within $15 \mathrm{~mm}$ from the pleura), which were the right posteroinferior subpleural area, right posterosuperior subpleural area, right anterior subpleural area, and right medial subpleural area, and the last one was placed in the right central area $20 \mathrm{~mm}$ away from the pleura (Fig. S1). The number of lobes with pulmonary opacities was also recorded and scored from 0 to 5 , with 5 indicating all 5 lobes were involved.

4. Dynamic evolution: Dynamic changes of volume, density, and frequency of opacities by location from symptom onset (day 1) to beyond day 15 were investigated.

\section{Clinical and laboratory characteristics}

Demographic information, exposure type, initial symptoms (fever, cough, sputum, shortness of breath, dyspnea, anorexia, or diarrhea), possible exposure time, time of initial symptoms, date of admission to hospital, comorbidity, and clinical laboratory findings including arterial oxygen saturation $\left(\mathrm{SaO}_{2}\right)$, blood cell counts (blood leukocyte count, lymphocyte count, and platelet count), and biomarkers of inflammation (C-reactive protein level, procalcitonin level, lactate dehydrogenase), hepatic and renal function (aspartate aminotransferase level, alanine aminotransferase level, creatinine level), and coagulation (D-dimer level) were collected.

\section{Statistical analysis}

All consecutive data were listed as means \pm standard deviations (SDs) or medians with interquartile ranges (IQRs) for Gaussian and skewed distributed data. Skewed distributed data were tried to convert to Gaussian distribution by logarithmic transformation for variance analysis. The Chi-square test, Kruskal-Wallis test, or Mann-Whitney test was used to examine statistical differences for ratio and skewed distributed data. All statistical analyses were performed using the $\mathrm{R}$ statistical software version 3.0.3.

\section{Results}

A total of 626 patients diagnosed with COVID-19 from 24 designated hospitals in Jiangsu Province were initially recruited to this study. One hundred and forty-two patients were excluded from the study because of either no clinical records or missing or incomplete chest CT images, or images with poor quality (Fig. 1). The remaining 484 patients from 21 hospitals (3 were excluded) were assigned to three severity 
groups (asymptomatic/mild, $n=63$; moderate, $n=378$; severe/critically ill, $n=43$ ). Baseline characteristics are shown in Table 1. There was marked disparity in median age between the three groups, manifesting as an increased age with increased severity $(p<0.001)$. Fever (up to $83.7 \%$ ) and cough/sputum (up to $69.8 \%$ ) were the most common symptoms in symptomatic patients. Analyses of laboratory test results for $\mathrm{SaO}_{2}$, blood leukocyte count,

Table 1 Baseline characteristics of study patients

\begin{tabular}{|c|c|c|c|c|}
\hline \multirow[t]{2}{*}{ Characteristics } & \multicolumn{4}{|l|}{ Disease severity } \\
\hline & Asymptomatic/mild ( $n=63,13.0 \%)$ & Moderate $(n=378,78.1 \%)$ & Sever/critically ill $(n=43,8.9 \%)$ & $p$ value \\
\hline Age (years) & $33(22,51)$ & $46(34,56)$ & $59(52,71)$ & $<0.001$ \\
\hline Gender, male & $31 / 63(49.2 \%)$ & $202 / 378(53.4 \%)$ & $27 / 43(62.8 \%)$ & 0.377 \\
\hline Incubation period (days) & $8(5,11)$ & $6(3,10)$ & $5(3,8)$ & 0.125 \\
\hline \multicolumn{5}{|l|}{ Initial symptoms } \\
\hline Fever & $14 / 30(46.7 \%)^{\mathrm{c}}$ & $278 / 378(73.5 \%)$ & $36 / 43(83.7 \%)$ & $<0.001$ \\
\hline Cough/sputum & $19 / 30(63.3 \%)^{\mathrm{c}}$ & $224 / 378(59.3 \%)$ & $30 / 43(69.8 \%)$ & $<0.001$ \\
\hline Shortness of breath/dyspnea & $0 / 30(0 \%)^{\mathrm{c}}$ & $10 / 378(2.7 \%)$ & $5 / 43(11.6 \%)$ & 0.007 \\
\hline Comorbidity $^{\mathrm{a}}$ & $8 / 63(12.7 \%)$ & $76 / 378(20.1 \%)$ & $16 / 43(37.2 \%)$ & 0.008 \\
\hline \multicolumn{5}{|l|}{ Exposure type } \\
\hline Wuhan exposure & $18 / 63(28.6 \%)$ & $148 / 378(39.2 \%)$ & $16 / 43(37.2 \%)$ & 0.275 \\
\hline COVID-19 patients exposure & $34 / 63(54.0 \%)$ & $139 / 378(36.8 \%)$ & $12 / 43(27.9 \%)$ & 0.012 \\
\hline Contact history $^{\mathrm{b}}$ & $8 / 63(12.7 \%)$ & $40 / 378(10.6 \%)$ & $8 / 43(18.6 \%)$ & 0.284 \\
\hline No exposure & $3 / 63(4.8 \%)$ & $51 / 378(13.5 \%)$ & $7 / 43(16.3 \%)$ & 0.116 \\
\hline \multicolumn{5}{|l|}{ Type of disease } \\
\hline Single onset & $10 / 63(15.9 \%)$ & $199 / 378(52.6 \%)$ & $23 / 43(53.5 \%)$ & $<0.001$ \\
\hline Clustering onset & $53 / 63(84.1 \%)$ & $179 / 378(47.4 \%)$ & $20 / 43(46.5 \%)$ & $<0.001$ \\
\hline \multicolumn{5}{|l|}{ Laboratory tests } \\
\hline $\mathrm{SaO}_{2}(\%)$ & $98.2(98.0,98.4)$ & $97.9(96.4,99.0)$ & $95.3(93.2,97.3)$ & $<0.001$ \\
\hline Blood leukocyte count $\left(\times 10^{\wedge} 9 / \mathrm{L}\right)$ & $5.3(4.5,6.6)$ & $4.8(3.8,6.0)$ & $4.2(3.5,5.9)$ & 0.033 \\
\hline$<4 \times 10^{\wedge} 9 / \mathrm{L}$ & $9 / 57(15.8 \%)$ & $93 / 309(30.1 \%)$ & $14 / 37(37.8 \%)$ & 0.040 \\
\hline$>10 \times 10^{\wedge} 9 / \mathrm{L}$ & $1 / 57(1.8 \%)$ & $5 / 309(1.6 \%)$ & $3 / 37(8.1 \%)$ & 0.077 \\
\hline Lymphocyte count $\left(\times 10^{\wedge} 9 / \mathrm{L}\right)$ & $1.8(1.3,2.5)$ & $1.3(1.0,1.7)$ & $0.6(0.5,0.9)$ & $<0.001$ \\
\hline$<1.5 \times 10^{\wedge} 9 / \mathrm{L}$ & $18 / 54(33.3 \%)$ & $195 / 318(61.3 \%)$ & $36 / 37(97.3 \%)$ & $<0.001$ \\
\hline Platelet count $\left(\times 10^{\wedge} 9 / \mathrm{L}\right)$ & $216(164,263)$ & $182(152,212)$ & $154(120,191)$ & $<0.001$ \\
\hline$<150 \times 10^{\wedge} 9 / \mathrm{L}$ & $9 / 58(15.5 \%)$ & $71 / 322(22.0 \%)$ & $15 / 37(40.5 \%)$ & 0.014 \\
\hline C-reactive protein $(\geq 10 \mathrm{mg} / \mathrm{L})$ & $4 / 50(8.0 \%)$ & $143 / 300(47.7 \%)$ & $19 / 31(61.3 \%)$ & $<0.001$ \\
\hline Procalcitonin $(\geq 0.5 \mathrm{ng} / \mathrm{mL})$ & $5 / 50(10.0 \%)$ & $55 / 295(18.6 \%)$ & $6 / 27(22.2 \%)$ & 0.274 \\
\hline Lactate dehydrogenase $(\geq 250 \mathrm{U} / \mathrm{L})$ & $6 / 37(16.2 \%)$ & $103 / 232(44.4 \%)$ & $17 / 26(65.4 \%)$ & $<0.001$ \\
\hline Aspartate aminotransferase $(>40 \mathrm{U} / \mathrm{L})$ & $4 / 48(8.3 \%)$ & $71 / 270(26.3 \%)$ & $13 / 30(43.3 \%)$ & 0.002 \\
\hline Alanine aminotransferase $(>40 \mathrm{U} / \mathrm{L})$ & $6 / 46(13 \%)$ & $70 / 273(25.6 \%)$ & $8 / 30(26.7 \%)$ & 0.170 \\
\hline D-dimer $(\geq 0.5 \mathrm{mg} / \mathrm{L})$ & $11 / 54(20.4 \%)$ & $75 / 311(24.1 \%)$ & $17 / 36(47.2 \%)$ & 0.007 \\
\hline Creatine $(\mu \mathrm{mol} / \mathrm{L})$ & $63(47,79)$ & $63(50,78)$ & $62(51,82)$ & 0.975 \\
\hline ICU /IMV/CRRT/ECMO & $1 / 63(1.6 \%)$ & $13 / 378(3.4 \%)$ & $15 / 43(34.9 \%)$ & $<0.001$ \\
\hline
\end{tabular}

Categorical variables are expressed as number (percentage) while continuous variables are described as median and interquartile range

$I C U$ intensive care unit, IMV invasive mechanical ventilation, CRRT continuous renal replacement therapy, ECMO extracorporeal membrane oxygenation

${ }^{a}$ Comorbidity include hypertension, coronary heart disease, cardiac dysfunction III-IV, poor physical fitness (unable to climb stairs, do daily housework, etc.), gastrointestinal hemorrhage caused by cirrhosis, hepatic encephalopathy and portal hypertension, diabetes, chronic kidney dysfunction, dialysis, malignant tumor, hematological malignancies, connective tissue disease, and stroke

${ }^{\mathrm{b}}$ Contact history refers to those patients who had contact with people from Wuhan before the onset of disease

${ }^{\mathrm{c}}$ A total of 33 patients were asymptomatic in the asymptomatic/mild group and were excluded when calculating the symptom percentage 
lymphocyte count, and platelet count showed downward trends from asymptomatic/mild to severe/critically ill. Conversely, C-reactive protein, lactate dehydrogenase, aspartate aminotransferase, and D-dimer concentrations above the normal range $(\geq 10 \mathrm{mg} / \mathrm{L}, \geq 250 \mathrm{U} / \mathrm{L},>40 \mathrm{U} /$ $\mathrm{L}$ and $\geq 0.5 \mathrm{mg} / \mathrm{L}$, respectively) tended to be more common in patients with severe disease. Patients with severe disease tended to have more adverse events including admission to ICU, invasive mechanical ventilation, continuous renal replacement therapy, and extracorporeal membrane oxygenation $(34.9 \%, p<0.001)$.

\section{Imaging characteristics by severity}

A total of $954 \mathrm{CT}$ scans were performed in the 484 patients (asymptomatic/mild 122 [12.8\%], moderate 747 [78.3\%], severe/critically ill 85 [8.9\%]). Numbers of CT scans by disease severity and time points are illustrated in Fig. S2. The imaging characteristics are shown in Table 2. Among the asymptomatic/mild group, $14.8 \%$ of CT scans showed no abnormal findings, and this was significantly greater than for the other two groups $(p<0.001)$. When pooling CT scans at different time points together, volume, density, and location demonstrated significant differences between the three groups. The severe/critically ill group showed the lowest total lung volume and aerated lung and greatest volume of pulmonary opacities with $3548.7 \mathrm{~mL}, 2557.0 \mathrm{~mL}$, and $491.4 \mathrm{~mL}$, respectively. For different components and locations of opacities, volume of consolidation was more than GGO and volume of opacities in the lower lungs were more than that in the upper lungs. Declines were found in attenuation as disease severity decreased.

Heterogeneous distribution of the pulmonary opacities was observed in all groups. The most common location for opacities was the subpleural area of posteroinferior lung (Fig. S3), most prominently in the severe/critically ill group with frequency of $45.3 \%$, followed by the subpleural area of posterosuperior lung $(32.6 \%)$; the

Table 2 Computed tomography (CT) results in patients with COVID-19, according to disease severity

\begin{tabular}{|c|c|c|c|c|}
\hline \multirow[t]{2}{*}{ Characteristics } & \multicolumn{4}{|l|}{ Disease severity } \\
\hline & Asymptomatic/mild ( $n=63,13.0 \%)$ & Moderate $(n=378,78.1 \%)$ & Severe/critically ill $(n=43,8.9 \%)$ & $p$ value \\
\hline Number of chest CT examinations & $122 / 954(12.8 \%)$ & $747 / 954(78.3 \%)$ & $85 / 954(8.9 \%)$ & - \\
\hline $\begin{array}{l}\text { Number of CT examinations without } \\
\text { pulmonary opacity }\end{array}$ & $18 / 122(14.8 \%)$ & $15 / 747(2.0 \%)$ & $0 / 85(0 \%)$ & $<0.001$ \\
\hline $\begin{array}{l}\text { Number of CT examinations with slice } \\
\text { thickness } \leq 3 \mathrm{~mm}\end{array}$ & $114 / 122(93.4 \%)$ & $654 / 747(87.6 \%)$ & $72 / 85(84.7 \%)$ & 0.108 \\
\hline \multicolumn{5}{|l|}{ Volume $(\mathrm{mL})$} \\
\hline Whole lung & $4637.4(3687.7,5724.5)$ & $4429.7(3575.9,5338.5)$ & $3548.7(2538.3,4393.6)$ & $<0.001$ \\
\hline Aerated lung & $4636.0(3585.6,5647.4)$ & $4216.6(3307.1,5094.7)$ & $2557.0(1921.0,3921.7)$ & $<0.001$ \\
\hline Pulmonary opacities & $1.7(0.1,44.9)$ & $129.8(34.0,302.5)$ & $491.4(199.0,1041.0)$ & $<0.001$ \\
\hline Ground-glass opacities & $0.1(0,5.7)$ & $9.1(1.2,32.5)$ & $14.8(0.9,66.4)$ & $<0.001$ \\
\hline Consolidation & $0.4(0,20.3)$ & $97.5(16.8,238.8)$ & $437.3(134.2,977.8)$ & $<0.001$ \\
\hline Opacities in the upper lobes & $0(0,1.8)$ & $22(0.7,98.7)$ & $227.7(46.6,496.2)$ & $<0.001$ \\
\hline Opacities in the lower lobes & $0(0,28.7)$ & $76.2(13.8,190.1)$ & $288.3(102.4,554.6)$ & $<0.001$ \\
\hline \multicolumn{5}{|l|}{ Density (X-ray attenuation, $\mathrm{Hu}$ ) } \\
\hline Whole lung & $-847(-864,-821)$ & $-826(-856,-786)$ & $-750(-802,-684)$ & $<0.001$ \\
\hline Pulmonary opacities & $-602.5(-713,-484)$ & $-634(-711,-540)$ & $-576(-666,-523)$ & 0.003 \\
\hline Opacities in the upper lobes & $-656(-736,-566)$ & $-676(-744,-577)$ & $-634(-696,-560)$ & 0.009 \\
\hline Opacities in the lower lobes & $-586(-689,-479)$ & $-624(-708,-518)$ & $-534(-645,-443)$ & $<0.001$ \\
\hline \multicolumn{5}{|c|}{ Frequencies of opacity by location $(\%)$} \\
\hline Right posteroinferior subpleural area & $4.7(2.4,7.1)$ & $20.5(18.6,22.6)$ & $45.3(43.0,48.8)$ & $<0.001$ \\
\hline Right posterosuperior subpleural area & $1.6(0.8,1.6)$ & $10.8(8.5,12.6)$ & $32.6(29.1,34.9)$ & $<0.001$ \\
\hline Right anterior subpleural area & $1.6(1.6,3.1)$ & $5.3(4.4,6.6)$ & $24.4(20.9,26.7)$ & $<0.001$ \\
\hline Right medial subpleural area & $1.6(1.6,1.6)$ & $4.1(3.5,5.2)$ & $22.1(19.8,26.7)$ & $<0.001$ \\
\hline Right central area & $1.6(0.8,1.6)$ & $4.9(4.5,5.5)$ & $26.7(23.3,27.9)$ & $<0.001$ \\
\hline Lung lobes involved & $3(1,4)$ & $5(3,5)$ & $5(5,5)$ & $<0.001$ \\
\hline
\end{tabular}

Variables are described as number (percentage) or median and interquartile range. Upper lobes include left upper lobe, right upper lobe, and right middle lobe; Lower lobes include left lower lobe and right lower lobe 
other areas (anterior and medial subpleural areas, central area) were comparable with frequencies of 22.1 to $26.7 \%$. The frequencies dropped with decreased disease severity, in all locations of the lung (Table 2 and Fig. 2).

In patients in the severe/critically ill group, significantly more lung lobes were involved compared with the other two groups, with the median number of lobes of 5 (5 to 5 ), 5 (3 to $5)$, and 3 ( 1 to 4$)$, respectively $(p<0.001)$.

\section{Dynamic evolution of imaging characteristics}

The three groups showed different progressive and regressive imaging patterns (see supplemental video).

The opacity volume was the greatest in the severe/critically ill group during the whole study period (Fig. 3a). Time series showed that opacity volumes (mainly for consolidation) continued to increase in the severe/critically ill group, reaching $30.3 \%$ of the whole lung beyond day 15 . However, for the moderate group, opacity volume maintained a steady state after days 13-15, accounting $7.7 \%$ of the whole lung. In the asymptomatic/mild group, the highest opacity volume was on days 1-3 (mean $1.9 \%$, 95\% confidence intervals [CI] 3.2\%), which dropped gradually to the lowest level beyond day 15 (mean $0.9 \%, 95 \%$ CI $0.3 \%$ ) (Fig. 3b). When classifying the opacities into GGO and consolidation, a remarkable decrease trend of GGO was observed compared with the increase trend of consolidation in the severe/critically ill group.

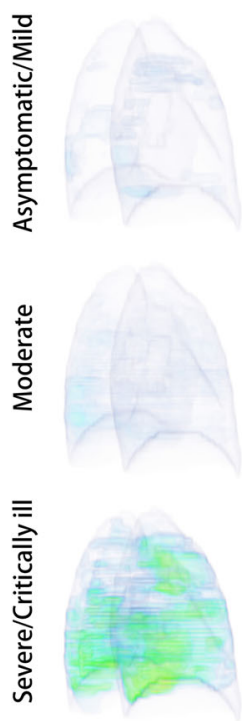

day $1-3$
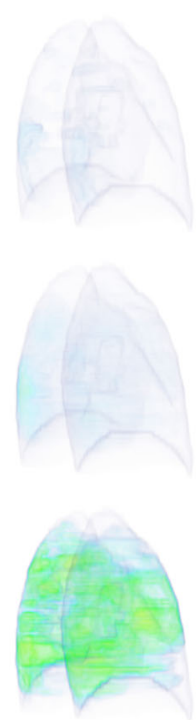

day 4-7

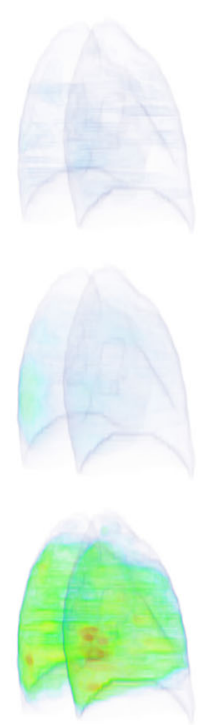

day $7-9$
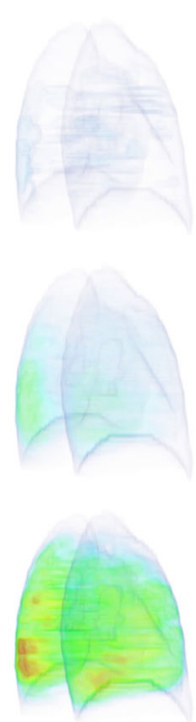

day $10-12$
Fig. 2 Three-dimensional heat maps show the frequency of location of pulmonary opacities in COVID-19 from the onset of symptoms (day 1) to beyond day 15. Asymptomatic/mild patients have trace opacities, resolving partially after 15 days (top row). Moderately ill patients have more opacities and peaked on days $13-15$, predominantly located in the
In the moderate group, the attenuation showed a trend of increase in the initial 9 days after symptom onset and decrease after day 12. Overall median attenuation in the severe/critically ill group was significantly higher than in the moderate group ( $-576 \mathrm{HU}$ vs $-634 \mathrm{HU}, p=0.002$ ). Opacities in the lower lobes had higher attenuation than that in the upper lobes $(-534 \mathrm{HU}$ vs $-634 \mathrm{HU}$ for the severe/critically ill group, $-624 \mathrm{HU}$ vs $-676 \mathrm{HU}$ for the moderate group, $p<0.001)$. An increase trend of attenuation was perceived beyond day 15 in the severe/ critically ill group, compared with continuous decline in the moderate group (Figs. 3c and 4).

Change of opacity frequency by day synchronized with the volume, most evidently in the posteroinferior subpleural area, reaching $61.6 \%$ in the severe/critically ill group, verses $25.1 \%$ and $5.5 \%$ in the moderate and the asymptomatic/mild groups, respectively (Figs. 3d and 5).

\section{Discussion}

In this province-based multicenter study, we have described CT imaging patterns and dynamic evolution in patients with COVID-19, according to disease severity. Jiangsu, an eastern Chinese province, with an area of 40,000 square miles and a population of about 80 million (ranked fifth in China provinces), is a representative district, and our findings may be of relevance to many other countries. The low death toll (no deaths in this study) and
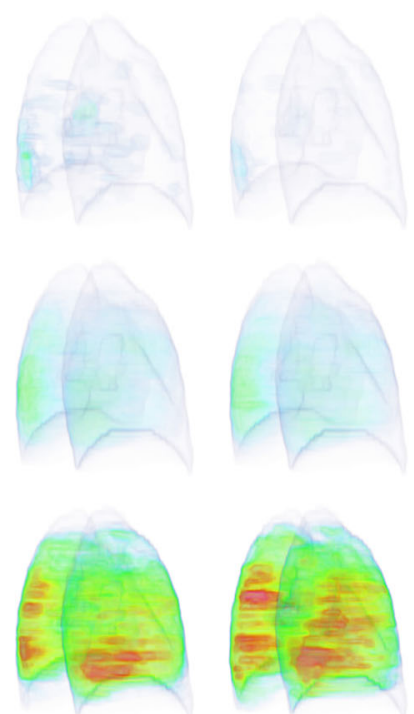

day $13-15$

beyond day 15
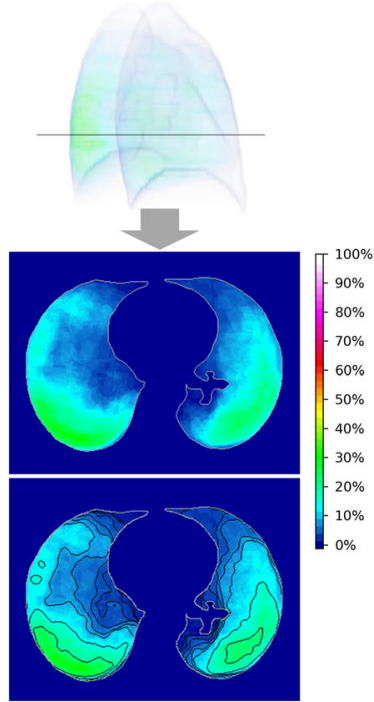

Frequency map with contour lines

posteroinferior subpleural area (middle row). Severe/critically ill patients have the most prominent opacities and continue to progress beyond day 15 (bottom row). Axial frequency map shows a typical pattern of posterior subpleural distribution of the opacities with decreased frequency in the anterior and medial subpleural areas 
high proportion of patients with moderate disease severity due to COVID-19 observed in our study are similar to reports from many other countries [2] and may indicate the common nature of COVID-19.

The demographics and clinical/laboratory findings in our study are also comparable with previously reported data $[3,8$, 11]. Severe cases tended to be older; had a shorter incubation period; had more comorbidities, abnormal blood cell counts, abnormal inflammatory biomarkers, and liver dysfunction; and experienced more serious adverse events. The early $\mathrm{CT}$ findings ( 3 days after onset) in this study were characterized as predominately subpleural GGO and posterior basal predominance for patients with different disease severity, consistent with previous studies $[6,12]$.

There was a tendency of subpleural distribution for pulmonary opacities, consistent with the underlying pathogenic pattern that distal bronchioles as well as alveolar epithelial cells impaired in viral pneumonia $[13,14]$. The posterior basal predominance shown in the heat map may due to the predilection of atelectasis posteriorly, corresponding to heterogeneous pulmonary opacity distribution in patients with acute respiratory distress syndrome [15]. Among all subpleural
Fig. 3 a-d CT measurements changing by day. a In the severe/ critically ill group, volume of aerated lung decreased while pulmonary opacities increased by day. b Volume of consolidation kept growing beyond 15 days in the severe/critically ill group, while peaked on days 13-15 in the moderate group. $\mathbf{c}$ The density of opacities dropped after day 12 in the moderate group; opacities in lower lobes had higher attenuation than those in upper lobes. d Posteroinferior subpleural area was the most commonly involved location in the lung, and the variation trend was similar to volume change in $\mathbf{b}$
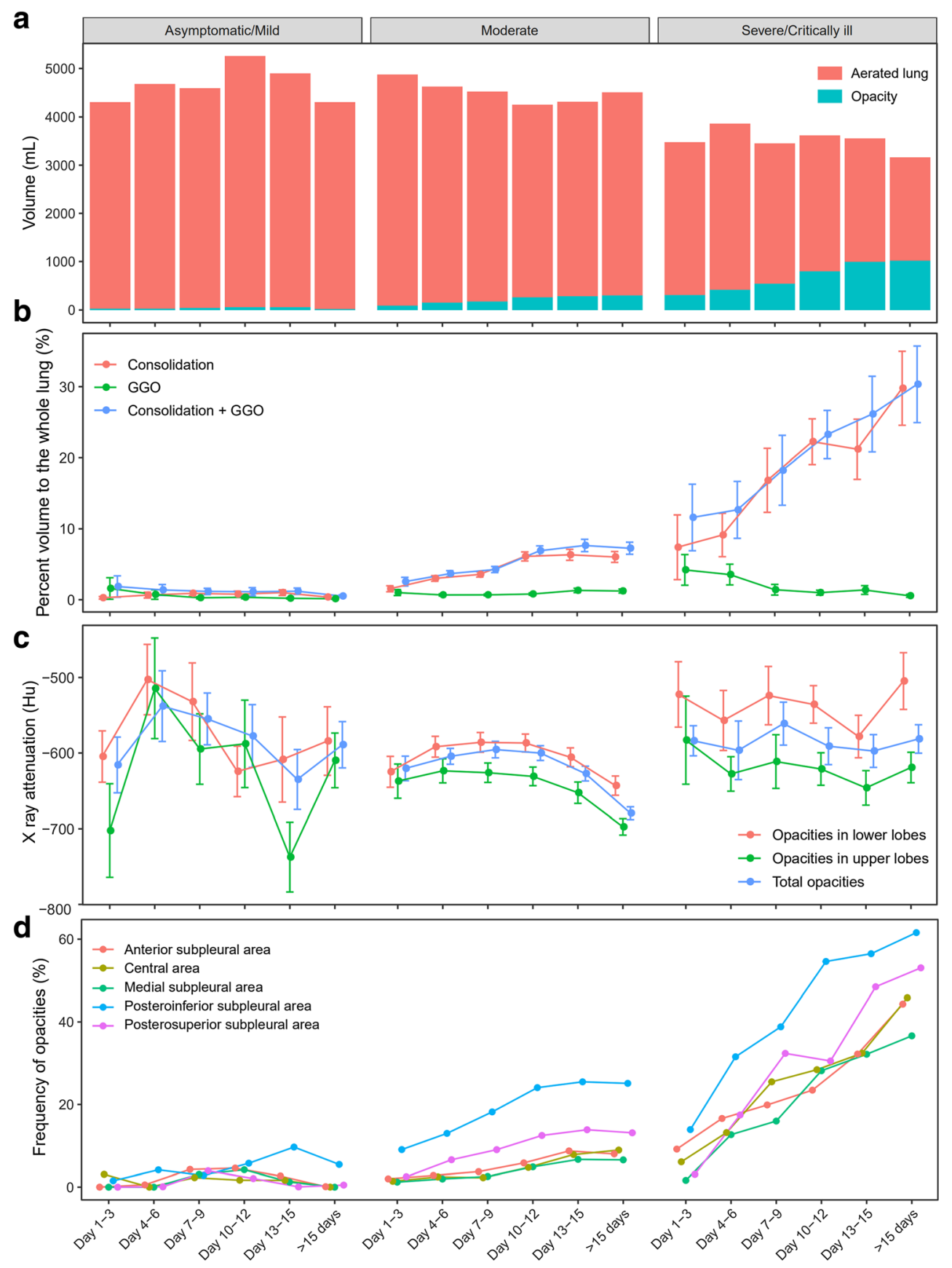
Fig. 4 A 64-year-old male diagnosed with moderate COVID-19. Non-contrast chest CT were performed on days 5,8 , 10 , and 15 after the onset of initial symptoms (a-d). The pulmonary opacities kept similar volume while the density decreased heterogeneously on day 15 compared with day 10

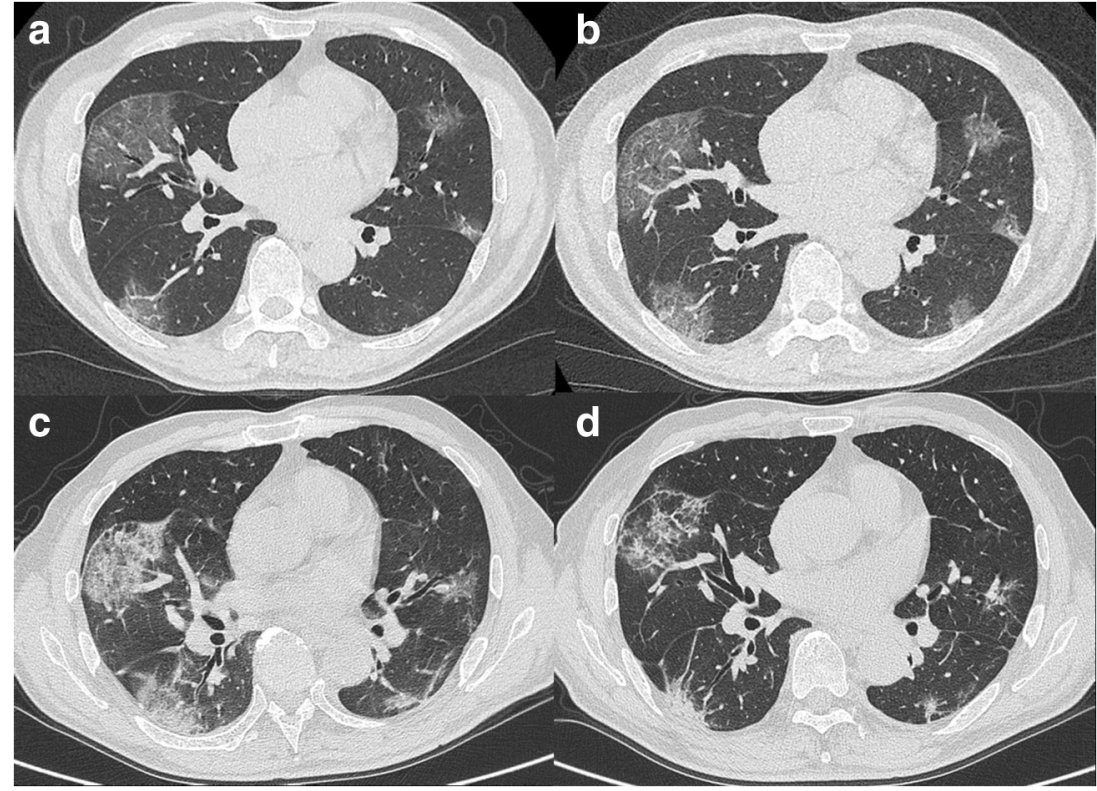

areas, the posteroinferior area of the lung showed leading frequency of opacity distribution as indicated in Fig. 3d, which may due to the anatomical characteristics of the bronchus: more vertical for lower lobes compared with the other lobes. This makes the lower lobes more susceptible to pathogen attack [8]. Our study further demonstrated a discrepancy in subpleural distribution which has not been elaborated in previous studies: the medial and anterior subpleural areas were less frequently involved compared with the posterior subpleural areas.

Part of the pulmonary opacities presented as GGO in this study, especially in the early stages of disease. This finding is consistent with several studies [8, 10, 16-18]. Although our understanding of the pathological mechanisms and effects of this specific novel coronavirus is limited, there is some evidence to explain the underlying causes of these radiological manifestations. In a study of two patients with lung adenocarcinoma surgery superimposed with COVID-19, damage of alveoli, together with proteinaceous and fibrous exudation, accounted for the early appearance of GGO on chest CT [19]. In studies of severe acute respiratory syndrome (SARS), focal lung injury was related to early radiologic findings [20]. As SARS and COVID-19 are both caused by coronavirus, it is possible that some of their pathological process overlap [21]. As the disease progresses, more inflammatory cells infiltrate the alveoli and interstitial space, followed by
Fig. 5 A 56-year-old female diagnosed with severe COVID-

19. Non-contrast chest CT were performed on days $5,11,14$, and 17 after the onset of initial symptoms (a-d). The bilateral subpleural opacities progressed to consolidation on day 17

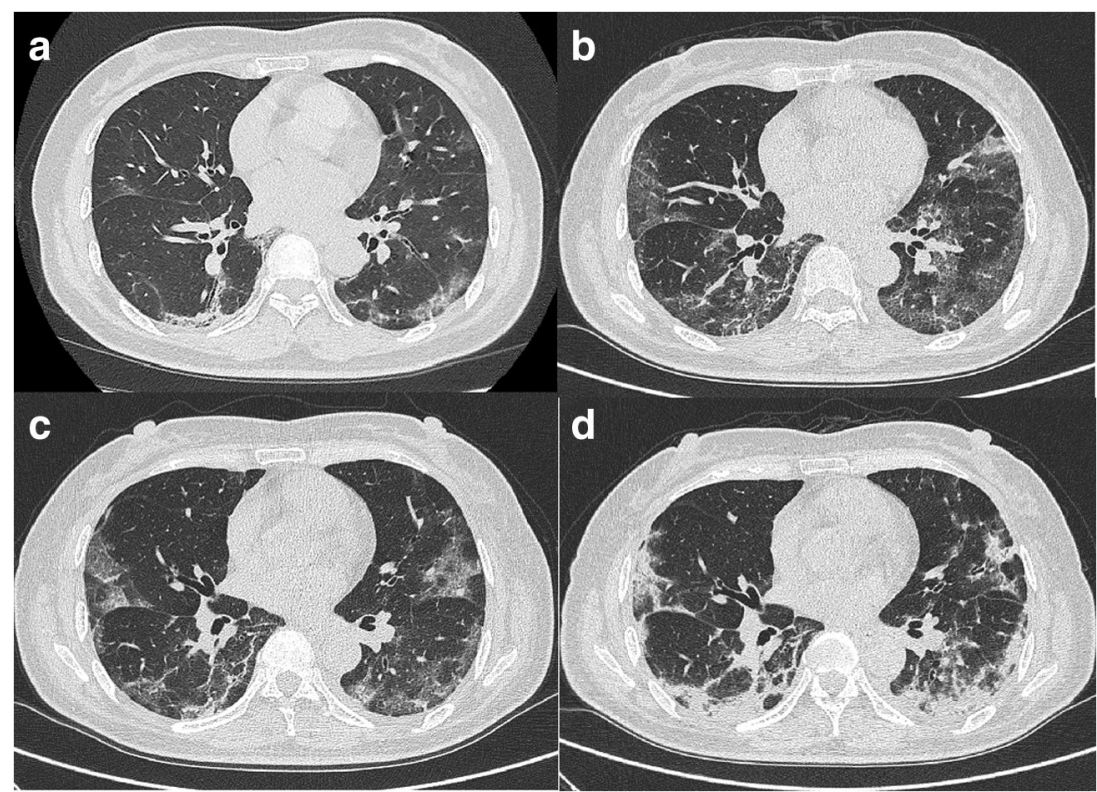


diffuse alveolar damage and hyaline membrane formation [22], which result in an appearance of consolidation on CT examination.

As for the dynamic evolution, a study of 63 COVID-19 patients from Wuhan showed an increased range of GGO and consolidation in 3-14 days after onset of symptoms [17]. Pan et al identified a peak stage of 9-13 days for lung involvement after symptom onset in 21 patients [10], compared with 6-11 days from another study of 90 patients [9]. In our study, we used an AI algorithm to automatically and quantitatively measure the percentage of opacity volume in 484 patients, which is different from scoring system based on visual evaluation in previous studies $[9,10,17]$. We concluded a peak stage of 13-15 days for opacity volume after symptom onset in moderately ill patients - the large majority in this study. Notably, for severe/critically ill patients, this peak stage extended to beyond 15 days, while for mild/ asymptomatic patients, the peak stage was just at the first 3 days after symptom onset. In severe/critically ill patients, different evolution patterns for GGO and consolidation were observed. There was a trend that GGO reduced continuously along with consolidation aggregation, suggesting a transformation of GGO to consolidation, during the course of disease. Varied patterns of imaging evolution are particularly useful in dynamically evaluating disease severity.

AI has been developed fast in recent years and has already been used to distinguish pneumonia in COVID-19 from community-acquired pneumonia [23]. The preliminary results are promising, but it should be noted the pre-test probability of COVID-19 varied with cities as well as countries, and the positive predictive value of $\mathrm{CT}$, even combined with $\mathrm{AI}$, may low in areas with low pre-test probability [24]. It should be avoided to overstate the role of AI in the diagnosis of COVID-19. In this study, however, we did not use AI to diagnose COVID-19, but for pulmonary lobe segmentation, lesion extraction, and $\mathrm{GGO} /$ consolidation labeling, these techniques are relatively mature. And the accuracy was verified in the study.

Our retrospective study has several limitations. Firstly, prospective studies of CT examination and patterns of evolution should be undertaken and validated in more institutions. Secondly, the relatively small sample size of mild/ asymptomatic cases resulted in relatively large variation in certain measurements. Consequently, although trends for some changes were observed, they did not reach statistical significance. Therefore, further studies that include more patients with asymptomatic/mild disease cases are required to strengthen the statistical power. Thirdly, opacity changes on CT were still progressing in the severe/critically ill patient group at the end of the study period (beyond day 15). Therefore, further studies are required to prolong the followup period to determine the potential break point for these patients. Finally, since CT findings were significantly different between the severity groups, we hypothesize that a combined CT score could be developed to classify disease severity, and predict disease outcome at an early stage. We plan to undertake this work in the near future.

\section{Conclusion}

In this multicenter study of patients with COVID-19, we have shown that pulmonary opacities vary in volume, density, and location, as well as the dynamic evolution, according to disease severity. Our findings provide insight into the nature of the disease and are potentially valuable in the evaluation and monitoring of the disease.

Funding information This study has received funding by the Ministry of Science and Technology of the People's Republic of China (2020YFC084370067).

\section{Compliance with ethical standards}

Guarantor The scientific guarantor of this publication is Shenghong Ju.

Conflict of interest The authors of this manuscript declare no relationships with any companies whose products or services may be related to the subject matter of the article.

Statistics and biometry No complex statistical methods were necessary for this paper.

Informed consent Written informed consent was waived by the Institutional Review Board.

Ethical approval Institutional Review Board approval was obtained.

Methodology

- retrospective

- observational

- multicenter study

\section{References}

1. Zu ZY, Jiang MD, Xu PP et al (2020) Coronavirus disease 2019 (COVID-19): a perspective from China. Radiology. https://doi.org/ 10.1148/radiol.2020200490

2. World Health Organization (2020) Coronavirus disease 2019 (COVID-19) situation report - 65. World Health Organization. Available via https:/www.who.int/emergencies/diseases/novelcoronavirus-2019. Accessed 25 Mar 2020

3. Guan W, Ni Z, Hu Y et al (2020) Clinical characteristics of coronavirus disease 2019 in China. N Engl J Med 382(18):1708-1720

4. Jiangsu Commission of Health Confirmed cases of 2019 novel coronavirus. http://wjw.jiangsu.gov.cn/art/2020/2/19/art_7290_ 8976979.html. Accessed 25 Mar 2020

5. National Health Commission of the People's Republic of China Diagnosis and treatment program for new coronavirus infection (trial version 5). February 8, 2020. Available via http://www.nhc.gov.cn/ 
xcs/zhengcwj/202002/d4b895337e19445f8d728fcafle3e13a.shtml. Accessed 25 Mar 2020

6. Shi H, Han X, Zheng C (2020) Evolution of CT manifestations in a patient recovered from 2019 novel coronavirus (2019-nCoV) pneumonia in Wuhan, China. Radiology. https://doi.org/10.1148/radiol. 2020200269

7. Kanne JP (2020) Chest CT findings in 2019 novel coronavirus (2019-nCoV) infections from Wuhan, China: key points for the radiologist. Radiology. https://doi.org/10.1148/radiol.2020200241

8. Shi H, Han X, Jiang $\mathrm{N}$ et al (2020) Radiological findings from 81 patients with COVID-19 pneumonia in Wuhan, China: a descriptive study. Lancet Infect Dis 20:425-434

9. Wang Y, Dong C, Hu Y et al (2020) Temporal changes of CT findings in 90 patients with COVID-19 pneumonia: a longitudinal study. Radiology. https://doi.org/10.1148/radiol.2020200843

10. Pan F, Ye T, Sun P et al (2020) Time course of lung changes on chest CT during recovery from 2019 novel coronavirus (COVID19) pneumonia. Radiology. https://doi.org/10.1148/radiol. 2020200370

11. Huang C, Wang Y, Li X et al (2020) Clinical features of patients infected with 2019 novel coronavirus in Wuhan, China. Lancet 395:497-506

12. Lei J, Li J, Li X, Qi X (2020) CT imaging of the 2019 novel coronavirus (2019-nCoV) pneumonia. Radiology. https://doi.org/ 10.1148/radiol.2020200236

13. Ruuskanen O, Lahti E, Jennings LC, Murdoch DR (2011) Viral pneumonia. Lancet 377:1264-1275

14. Kim EA, Lee KS, Primack SL et al (2002) Viral pneumonias in adults: radiologic and pathologic findings. Radiographics $22 \mathrm{Spec}$ No:S137-49

15. Sheard S, Rao P, Devaraj A (2012) Imaging of acute respiratory distress syndrome. Respir Care 57:607-612

16. Huang P, Tianzhu Liu LH (2020) Use of chest CT in combination with negative RT-PCR assay for the 2019 novel coronavirus but high clinical suspicion. Radiology. https://doi.org/10.1148/radiol. 2020200330

17. Pan Y, Guan H, Zhou S et al (2020) Initial CT findings and temporal changes in patients with the novel coronavirus pneumonia (2019-nCoV): a study of 63 patients in Wuhan, China. Eur Radiol. https://doi.org/10.1007/s00330-020-06731-x

18. Xie X, Zhong Z, Zhao W, Zheng C, Wang F, Liu J (2020) Chest CT for typical 2019-nCoV pneumonia: relationship to negative RTPCR testing. Radiology. https://doi.org/10.1148/radiol. 2020200343

19. Tian S, Hu W, Niu L, Liu H, Xu H, Xiao SY (2020) Pulmonary pathology of early phase 2019 novel coronavirus (COVID-19) pneumonia in two patients with lung cancer. J Thorac Oncol S1556-0864(20):30132-30135

20. Paul NS, Roberts H, Butany J et al (2004) Radiologic pattern of disease in patients with severe acute respiratory syndrome: the Toronto experience. Radiographics 24:553-563

21. Liu J, Zheng X, Tong Q et al (2020) Overlapping and discrete aspects of the pathology and pathogenesis of the emerging human pathogenic coronaviruses SARS-CoV, MERS-CoV, and 2019nCoV. J Med Virol 92:491-494

22. Xu Z, Shi L, Wang Y et al (2020) Pathological findings of COVID19 associated with acute respiratory distress syndrome. Lancet Respir Med 2600:19-21

23. Li L, Qin L, Xu Z et al (2020) Artificial intelligence distinguishes COVID-19 from community acquired pneumonia on chest CT. Radiology. https://doi.org/10.1148/radiol.2020200905

24. Hope MD, Raptis CA, Shah A, Hammer MM, Henry TS (2020) A role for CT in COVID-19? What data really tell us so far. Lancet 395:1189-1190

Publisher's note Springer Nature remains neutral with regard to jurisdictional claims in published maps and institutional affiliations. 